\title{
Estimation of Methicillin Resistant Staphylococcus aureus Carriage among Patients' Visitors (Adults) in a Tertiary Care Hospital
}

\author{
T.M. Nandan ${ }^{1 *}$ (D) and Srujan Kumar Bondugula² (D) \\ ${ }^{1}$ Department of Microbiology, Sambhram Institute of Medical Sciences and Research, D.K. Halli Road, BEML \\ Nagar, K.G.F, Karnataka, India. ${ }^{2}$ PES Institute of Medical Sciences and Research, Kuppam, Chittoor Dist., Andhra \\ Pradesh, India.
}

\begin{abstract}
As community at large is also one of the potential habitats for emergence of multidrug resistant bacteria, exemplified by Methicillin Resistant Staphylococcus aureus(MRSA), due to widespread misuse of antibiotics at various levels including from non-human-healthcare sources like veterinary and poultry care along with unrestricted pharmacies, it was prudent to investigate the less trodden arena of the patients' visitor populace in hospitals, that along with patients, actually forms the interface between the two and could have been contributing to the overall ecologic burden of such organisms there, often, hardly being accounted for. Nasal swabs (160) were processed on $5 \%$ sheep blood agar for primary isolation of $S$. aureus and subsequently Cephoxitin disc diffusion test on unsupplemented Mueller Hinton Agar at $35^{\circ} \mathrm{C}$ was used for phenotypic detection of mecA mediated oxacillin resistance as described by CLSI standard to identify MRSAs irrespective of the two clinico-genotypes of HA-and CA-MRSAs, the margin between which being increasingly becoming blurred off late. Five MRSAs were isolated out of 160 swabs, although the S.aureus isolates were 28/160, to give the prevalence of nasal carriage of $3 \%$ among the patients' visitors (adults), which would form a major chunk that cannot be disregarded, given the complex dynamics of microbial colonization and infection. The present study throws light on the often ignored group of population as potential source for transmission of extreme drug resistant bacteria in healthcare settings and goes a long way in abrogating their notoriety as the only sources of harmful superbugs.
\end{abstract}

Keywords: Staphylococcus aureus, MRSA carriage, patients' visitors, sheep blood agar, un-supplemented Mueller Hinton agar, Cefoxitin disc diffusion test.

\footnotetext{
*Correspondence: drnandantmv@gmail.com; 9148432749.
}

(Received: 24 Septemeber 2019; accepted: 12 December 2019)

Citation: T.M. Nandan and Srujan Kumar Bondugula, Estimation of Methicillin Resistant Staphylococcus aureus Carriage among Patients' Visitors (Adults) in a Tertiary Care Hospital, J Pure Appl Microbiol., 2019; 13(4):2301-2307. https://doi.org/10.22207/ JPAM.13.4.45

(C) The Author(s) 2019. Open Access. This article is distributed under the terms of the Creative Commons Attribution 4.0 International License which permits unrestricted use, sharing, distribution, and reproduction in any medium, provided you give appropriate credit to the original author(s) and the source, provide a link to the Creative Commons license, and indicate if changes were made. 


\section{INTRODUCTION}

Methicillin resistant Staphylococcus aureus (MRSA) has increasingly been a cause for concern as a nosocomial pathogen in healthcare facilities around the world". "MRSA" has been the buzzword in almost all healthcare facilities even in India not only among the medical fraternity but also among the members of the allied health sciences like nurses and technicians. It has also knocked the doors of the general public by the increasingly responsible mass communication systems. Due to effort of the latter for at-last responding to the need and threat of the so called 'post antibiotic era', wherein hardly any antibiotic would be available to treat the most dreaded infectious diseases due to pan drug resistant bacteria including the current bacterium of interest along with its vancomycin intermediately sensitive and vancomycin resistant mutant strains (Vancomycin intermediately sensitive Staphylococcus aureus and Vancomycin resistant Staphylococcus aureus) among the ever increasing family of other extremely resistant Gram positive and Gram negative bacteria. Also, adequate evidence is now available to implicate not only the physician community at large, but the pharmacies, the veterinarians, personnel in animal husbandry and poultry, for this scenario, through decades of misuse and overuse of the magic bullets often through neglect and apathy towards the initial reports of bacterial drug resistance right from the cautious predictions of Sir. Alexander Fleming himself ${ }^{2}$.

No wonder, the general community at large and the life on earth have been inflicted by this no less insane attitude and practice. In spite of multiple causations, unfortunately, healthcare settings are increasingly being implicated and projected as the sole emanating sources of these drug resistant bugs to the community including even the most vigilant and preferred healthcare providers across the country. Thus, it is the need of the hour to delve into the less investigated arena of the population of the community that directly interacts with the healthcare inpatient facilities as part of direct or indirect 'care and courtesy' for the suffering patients, like their 'visitors' during the treatment periods. As adult visitors dominate such a populace, it is worthwhile screening them for harboring resistant bacteria of various types. As MRSA is among the forefront of such bugs, having known for its widespread presence exemplified by its community specific variant, CA-MRSA (community associated MRSA) as well, it is a natural target of interest. Moreover, along with its healthcare associated variant, the popularly prevalent carrier among the healthcare personnel and/or environment (health careassociated/ HA- MRSA), MRSA is implicated in healthcare associated infections of varied spectrum ranging from superficial skin and soft tissue infections like furuncles, carbuncles, to the deep seated abscesses at multitudes of systemic sites like lungs, blood, bones and brain ${ }^{1}$. Although HA-MRSA is considerably different from CA-MRSA, both clinically and microbiologically, there have been reports of emergence of the third variety, i.e., HA-CO-MRSA (health care-associated- community onset-MRSA) evidenced by genetic studies ${ }^{3}$. As inpatients are also exposed to community visitors on several occasions during their stay, the possibility of a fourth variety of "CA-HO-MRSA" (community associated- health care onset-MRSA) cannot be excluded as well. Although the need is obvious and even expressed by very few ${ }^{4}$, there have hardly been any such studies reported. Hence, the present study has been aimed at knowing the carriage rate of MRSA among patients' visitors, especially the adult populace irrespective of the two major clinico-genotypes in addition to the practical bacteriologic task of culturing and characterizing in the Microbiology laboratory.

\section{Selection of population of patients' visitors}

In the background of the high to very high prevalence of infections due to MRSA reported by several hospitals across the country and around the world ${ }^{5}$, it is imperative to stress upon more and more research pertaining to the further details of epidemiology including those often ignored ones as explored in this present study. The trend so far with respect to estimation of carriage of MRSA has been more often limited to screening the hospital personnel on the one hand and children and less often adult population of the community on the other hand. Although the former group along with the hospital environment may evidently contribute to the incidence of healthcare associated 
transmission of colonization of MRSA and the subsequent complication of infections during the inpatient stay period or beyond, the latter, in the absence of any contact with healthcare settings, do not necessarily do so. Of the population that may be considered as belonging to healthcare facilities, visitors or caretakers of inpatients form a substantial proportion who are directly related to the load of inpatients, i.e., at least one accompanying the admitted patient. These visitors form that part of the community at large who are at the community-healthcare facility interface (including patients). The visitors, as obvious, may be anybody from the patients' families, friends and colleagues from varied background and thus form a significant group of people from the community who interact with the hospital personnel and hospital environment. Thus, their presence and demeanor during their stay within a healthcare facility cannot be ignored. Reasons for ignoring such a significant group that has the potential to transmit drug resistant bugs like MRSA are unknown and not discussed about but can be probably perceived to be those related to human ethos. But in the tradeoff against multidrug resistant bugs, the contrary is true and it is morally justifiable to identify all the possible sources of deadly pathogens.

\section{Bacteriology for MRSA}

Several studies have used blood agar ${ }^{6,5}$ as one of the plating media for primary isolation of Staphylococcus aureus from the swabs. Catalase test, coagulase tests, Gram staining, mannitol fermentation test have been recommended for routine identification of $S$. aureus. Blood agar with Oxacillin was preferred over mannitol salt agar in one of the studies ${ }^{7}$. Cephoxitin disc diffusion test has been one of the screening methods for routine detection of MRSA recommended by $\mathrm{CLSI}^{8}$ (Clinical and Laboratory Standards Institute, USA) so also the latex agglutination test for PBP2a, or a plate containing $6 \mu \mathrm{g} / \mathrm{ml}$ of oxacillin in Mueller-Hinton agar supplemented with $4 \% \mathrm{NaCl}$. Unsupplemented Mueller Hinton Agar is preferred over other enriched media for reliable detection of mecA mediated resistance ${ }^{8}$. Cephoxitin mannitol salt agar ${ }^{9}$ with varying concentrations of cephoxitin has been used for selective isolation of MRSA. Detection of mecA gene by $\mathrm{PCR}^{3}$ (Polymerase Chain Reaction) has been the most ideal test for confirmation of MRSAs. Historically (Staphylococcal Cassette Chromosome) SCCmec types I, II, or III are present in HA-MRSA and Panton-Valentine leukocidin gene, SCCmec type IV or V genes are determinative of CA-MRSA.

The aim of the present study thus was to estimate nasal MRSA carriage among patients' visitors (adults) to a tertiary care hospital. The objectives were: 1 . To collect nasal swabs from adult visitors to inpatients of the hospital. 2. To characterize and identify MRSA by culture and sensitivity testing in Microbiology laboratory.

\section{MATERIALS AND METHODS Type of study \\ Observational cross-sectional study. \\ Study population}

Patients' visitors from the community to a rural tertiary care hospital. Sampling and processing were carried out from $20^{\text {th }}$ April 2017 to $20^{\text {th }}$ June 2017 for a period of 2 months from any adult visitors to the inpatients from the community. Prior Ethical Clearance was obtained from the Institutional Research and Ethics Committee. Written informed consents were obtained from the participants.

\section{Study setting}

A rural tertiary healthcare centre in Chittoor District of Andhra Pradesh, bordering the other two states of Karnataka and Tamil Nadu.

\section{Sampling method}

Random sampling was carried out from various wards of the hospital. Sample size: 160 for an estimated prevalence of $11 \%{ }^{10,11}$ with $95 \%$ confidence level and a precision of 0.05 .

\section{Inclusion criteria}

Any adult visitors from the community to inpatients $<48$ hours $^{3}$ of admission.

\section{Exclusion criteria}

Visitors who had stayed along with their in patients for $>48$ hours, visiting hospital staff, and children.

Collection of samples and bacteriologic processing

MRSA screening was performed using a single sterile moistened cotton tipped swab. With aseptic precautions, anterior nares were swabbed and immediately inoculated onto 5\% 
sheep blood agar plates and incubated at $37^{\circ} \mathrm{C}$ for 24-48 hours for isolation of Staphylococcus aureus $^{11}$. S. aureus isolates were identified by colony characteristics, Gram staining, catalase test, coagulase tests, and mannitol fermentation test. Golden yellow, moist, opaque, hemolytic or non-hemolytic colonies were selected and the culture smears were made for Gram staining. Typical Gram positive cocci in clusters about $1 \mu \mathrm{m}$ in size were presumptively identified as $S$. aureus. Tube coagulase and mannitol fermentation tests were put up, incubated overnight and the results were observed and recorded the next day. Mannitol motility medium was used for mannitol fermentation test. Appropriate subcultures were made for proper isolation of colonies whenever necessary for further characterization including on media not containing blood. Such colonies were subjected to catalase test with $3 \%$ hydrogen peroxide and looked for brisk effervescence. Cephoxitin resistance (surrogate for Oxacillin resistance ${ }^{8}$ ) of the $S$. aureus isolates was checked for by performing Kirby Bauer disc diffusion test on unsupplemented Mueller Hinton agar plates with incubation at $35^{\circ} \mathrm{C}$ for 24 hours and the zone diameters were measured. Any zone of inhibition $\geq 22 \mathrm{~mm}$ in diameter was considered 'susceptible' and that with $\leq 21 \mathrm{~mm}$ as 'resistant' to cephoxitin and hence as Methicillin Resistant S. aureus. ATCC 25923 S. aureus and ATCC 43300 MRSA were used as standard strains as appropriate for quality control at all the levels. They were utilized from the stock culture maintained in the NABL accredited laboratory after having procured from a commercial vendor. CLSI (Clinical laboratory standards institute) guidelines were referred for interpretation of zone diameters for cephoxitin susceptibility testing ${ }^{8}$.

\section{RESULTS}

Staphylococcal isolates from the nasal swabs of patients' visitors from various wards of the hospital have been depicted in Table 1. Overall percentage of MRSAs isolated was $3 \%$. Greater number of samples were collected from the ICU-OT (intensive care units-operation theater) complex (23\%), followed by Gynaecology $(18 \%)$, and the least number from Paediatrics wards (2\%). MRSAs were isolated from visitors in the Emergency ward (2), ICU-OT complex (2) and Labour Waiting Room (1). Although greater number of $S$. aureus was isolated in Gynaecology ward, no MRSAs were present. Similarly, less number of $S$. aureus/MRSA was isolated from Orthopaedics and General Surgery wards.

All the isolates that were phenotypically tested and identified during the conventional identification procedure have been shown in Table 2. Stapylococcus aureus (including MRSA) made up $18 \%$ of all the isolates. Coagulase negative staphylococci comprised the majority (34\%), immediately followed by Micrococci (31\%). Gram negative cocci were the least at $1 \%$.

As part of the standard identification protocol, in addition to catalase and coagulase tests, mannitol fermentation test was carried out on all S.aureus isolates and the result is shown in Table 3. All the 5 MRSA isolates fermented

Table 1. Ward-wise No., of samples and isolates

\begin{tabular}{lcccc}
\hline Ward & $\begin{array}{c}\text { No. of } \\
\text { samples }\end{array}$ & $\begin{array}{c}\text { Percentage } \\
\%\end{array}$ & $\begin{array}{c}\text { No. of } \\
\text { S.aureus }\end{array}$ & $\begin{array}{c}\text { No. of } \\
\text { MRSAs }\end{array}$ \\
\hline Emergency ward & 23 & 14 & 3 & 2 \\
General Medicine & 9 & 6 & 2 & 0 \\
Gynaecology & 28 & 18 & 7 & 0 \\
ICU-OT Complex & 37 & 23 & 6 & 2 \\
Labour Waiting & 23 & 14 & 4 & 1 \\
Room & 17 & 11 & 0 & 0 \\
Orthopaedics & 3 & 2 & 0 & 0 \\
Paediatrics & 20 & 13 & 1 & 0 \\
General Surgery & 160 & 100 & 23 & 5 \\
TOTAL & & & & (Overall 3\%) \\
& & & 2304 &
\end{tabular}


mannitol. One S.aureus isolate did not ferment mannitol.

Analyzing the age-group of the visitors who carried MRSA and the wards they were in, as in Table 4, it was found that three of the visitors were in their twenties, one in forties, and another one in sixties, younger ones being colonized more. ICU-OT complex, Emergency ward and Labour Waiting Room had all the burden of the superbug.

Further, out of the overall $3 \%$ prevalence of MRSA carriage among the visitors, $6 \%$ were males and 1\% was females as shown in Table 5.

Table 2. Distribution of isolates

\begin{tabular}{lcc}
\hline Isolate & No. & $\begin{array}{c}\text { Percentage } \\
\%\end{array}$ \\
\hline $\begin{array}{l}\text { Coagulase negative } \\
\text { staphylococci }\end{array}$ & 55 & 34 \\
Diphtheroids & 21 & 13 \\
Gram Negative Cocci & 2 & 1 \\
Gram Positive Bacilli & 4 & 3 \\
Micrococci & 50 & 31 \\
Staphylococcus aureus & 28 & 18 \\
TOTAL & 160 & 100 \\
\hline
\end{tabular}

Table 3. Mannitol fermentation among the isolates

\begin{tabular}{lcc}
\hline Isolates & No. & $\begin{array}{c}\text { Mannitol } \\
\text { fermentation } \\
+ \text { No, (\%) }\end{array}$ \\
\hline $\begin{array}{l}\text { Staphylococcus aureus } \\
\text { (including MRSAs) }\end{array}$ & 28 & $27(96.4)$ \\
$\begin{array}{l}\text { Staphylococcus aureus } \\
\text { (excluding MRSAs) }\end{array}$ & 23 & $22(96)$ \\
MRSA (18\% of S.aureus) & 5 & $5(100)$ \\
\hline
\end{tabular}

Table 4. Distribution of MRSA isolates: Age of visitors ward-wise

\begin{tabular}{lc}
\hline $\begin{array}{l}\text { Age in } \\
\text { years }\end{array}$ & Ward \\
\hline 27 & ICU-OT Complex \\
26 & ICU-OT Complex \\
45 & Emergency ward \\
22 & Emergency ward \\
65 & Labour Waiting Room \\
\hline
\end{tabular}

Table 5. Gender-wise prevalence of MRSA carriage

\begin{tabular}{lccc}
\hline Gender & $\begin{array}{c}\text { Total } \\
\text { No. }\end{array}$ & $\begin{array}{c}\text { No. of MRSA } \\
\text { isolates }\end{array}$ & $\begin{array}{c}\text { Percentage } \\
\%\end{array}$ \\
\hline Males & 69 & 4 & 6 \\
Females & 91 & 1 & 1 \\
Overall & 160 & 5 & $\mathbf{3}$ \\
\hline
\end{tabular}

\section{DISCUSSION}

Although the estimation of MRSA carriage in various population groups like healthcare workers has been one of the most popular topics of present day research, the present study on the sample population of patients' visitors from the community at large, is unique and not often explored.

The overall percentage and hence the prevalence of MRSA among the adult visitors to patients in various wards who fulfilled the inclusion criteria is $3 \%$ out of the total 160 sample swabs collected and processed in Microbiology laboratory. The finding that is complementary to the burden contributed by several other populations of collective importance like the (a) outpatients, (b) inpatients and (c) healthcare personnel as evident by many research studies pertaining to healthcare settings ${ }^{7}$, hence bears significance.

Nevertheless, the microbial isolates from human carriers in the general population represent those of the community at large as often in vogue. Hence, at least for now and till further research studies, the population represented by the present sample (d), which actually forms a discrete group at the interface of healthcare settings and the community, can be better compared with the studies restricted to the apparently healthy carrier members of the community like school children, etc.

Several methods have been employed for bacteriology of MRSA. A community based study on nasal carriage of Staphylococcus aureus by Chatterjee et al. ${ }^{12}$, on 489 school children of 5-15 year age group from various slums in the north of India, reported $3.89 \%$ of MRSA carriage similar to the present study. The selective mannitol salt agar and Oxacillin screen agar $(6 \mu \mathrm{g} / \mathrm{ml})$ with $4 \% \mathrm{NaCl}$ were used for that screening procedure. 
A study by Sharma $Y$ et al..$^{6}$, comprising of 200 healthy subjects attending outpatient departments and healthcare workers in Delhi, reported 5\% MRSA carriage in the anterior nares, slightly higher than that of the present study. The sample included adults and children from the community apart from the healthcare workers. Oxacillin $(1 \mu \mathrm{g})$ disc diffusion test was used for detection of MRSA.

Another study by Reta et al. ${ }^{13}$, on nasal carriage of MRSA in 300 school children in Ethiopia, Africa, a similar $6 \%$ prevalence was recorded. Oxacillin $(1 \mu \mathrm{g})$ and Cephoxitin (30 $\mu \mathrm{g})$ disc diffusion test was used for MRSA detection.

In the study involving 280 apparently healthy individuals in East Sikkim by Devjyoti et al. ${ }^{11}$, a prevalence of $11 \%$ was noted and was one of the highest recorded MRSA carriage rates. Mueller Hinton agar with $4 \% \mathrm{NaCl}$ and Oxacillin disc diffusion test were used.

A study in Puducherry, south of eastern India by Mathanraj S et al. ${ }^{7}$, involving 90 inpatients, 53 outpatients and 57 health care workers representing the hospital environment reported an overall $8.5 \%$ MRSA carriage rate which is not so different from that of the present study. The outpatient MRSA carriage rate was about $4 \%$ and among the healthcare workers, it was about $2 \%$.

Another study involving only healthcare personnel done in Tiruchirapalli, south of India, by Rajendran et al. $^{14}$, reported $6 \%$ of nasal MRSA carriage. Thus the target population of the present study forms a remarkable and discrete group that equally contributes to the burden of MRSA load in a given healthcare setting.

Several authors ${ }^{5,7}$ have recommended anterior nares as the sole site for sampling over the less convenient and less contributing sampling sites like the pharyngeal and axillary swabs. However perineal swabs do increase the sensitivity.

Younger age group has shown preponderance for MRSA carriage in the present study as well as documented by others ${ }^{14}$.

Though females are more prone to acquiring colonization with MRSA, more number of males $(6 \% ; 4 / 69)$ have carried MRSA in the present study in contrast to $1 \% ; 1 / 91$ among the females, similar to the one by Mathanraj et al. ${ }^{7}$, in Puducherry.
All MRSA strains in the present population fermented mannitol and thus the test may be possibly incorporated even for primary isolation in the form of complex media like mannitol salt agar or cephoxitin mannitol salt agar 7,9 .

Among the other bacterial isolates, Coagulase Negative Staphylococci have been isolated from $34 \%$ of the nasal swabs. It was beyond the scope of the present study to further characterize them. Given the fact that they are also known to harbour mecA gene, it is further justifiable of the selection of the target population of the present study. CONS is also known be highly prevalent as nasal colonizers as reported by other authors ${ }^{10}$ as well.

\section{CONCLUSION}

Patients' visitors (adults) to the rural tertiary care hospital from the community harbor Methicillin Resistant Staphylococcus aureus to a considerable degree and thus form one of the potential sources of this dreaded pathogen among others that could add on to the burden of healthcare associated infections. The prevalence is similar to that among the community at large \&healthcare personnel and hence opens up a new arena for regular surveillance activities involving this group for better infection control in all healthcare settings. The finding can go a long way in changing the deeply set notorious general opinion that healthcare facilities are the only major sources of multi-drug resistant bacteria in spite of decades of rampant misuse and abuse of antibiotics that has been taking place for human and other than human-healthcare-activities in the community. The study would thus try to enlighten us to plan better preventive strategies to tackle the global gradual slide into the post antibiotic era.

\section{ACKNOWEDGEMENTS}

The authors thank ICMR, the Principals and Heads of the Departments of Microbiology of PESIMSR, Kuppam, and SIMSAR, KGF, for their encouragement.

\section{CONFLICTS OF INTEREST}

The authors declare that there is no conflict of interest. 


\section{FUNDING}

This study was carried out as an STSproject-2017 granted by the Indian Council of Medical Research. Publication is funded by the authors ICMR STS ID: 2017-03655.

\section{AUTHORS' CONTRIBUTION}

TMN conceptualization, design, execution, compilation, editing, and correspondence for publication. SKB provides assistance in designing and execution.

\section{DATA AVAILABILITY}

The data collected and used for the study are available with the ICMR STS-2017 registry and with the corresponding author.

\section{ETHICS STATEMENT}

Prior ethical clearance was obtained from the Institutional Research and Ethics Committee. Written informed consents were obtained from the participants.

\section{REFERENCES}

1. Sangeeta J, Pallab R, Vikas M, Jyoti B, Chitnis DS, et al. Methicillin resistant Staphylococcus aureus (MRSA) in India: Prevalence \& susceptibility pattern. Indian J Med Res., 2013; 137: 363-369.

2. Fleming A. Nobel Lecture. 1945. Accessed on: $10 \mathrm{Dec}$ 2019. Available from: https://www.nobelprize.org/ prizes/medicine/1945/fleming/lecture.

3. Michael ZD, Robert SD. Community-associated methicillin-resistant Staphylococcus aureus: Epidemiology and clinical Consequences of an emerging epidemic. Clinical microbiology Reviews, 2010; 23(3): 616-687. https://doi.org/10.1128/ CMR.00081-09

4. David MZ, Siegel JD, Chambers HF, Daum RS Determining whether methicillin-resistant Staphylococcus aureus is associated with health care. JAMA, 2008; 299: 519. https://doi.org/10.1001/ jama.299.5.519-a

5. Gonsu KH, Kouemo SL, Toukam M, Ndze VN and Koulla SS. Nasal carriage of methicillin resistant Staphylococcus aureus and its antibiotic susceptibility pattern in adult hospitalized patients and medical staff in some hospitals in Cameroon. Journal of Microbiology and Antimicrobials, 2013; 5(3): 29-33. https://doi.org/10.5897/JMA2012.0232

6. Sharma Y, Jain S, Singh H, Govil V. Staphylococcus aureus: screening for nasal carriers in a community setting with special reference to MRSA. Scientifica, 2014; 2014: 479048, 1-5. https://doi.org/10.1155/2014/479048

7. Mathanraj S, Sujatha S, Sivasangeetha K, Parija SC. Screening for methicillin-resistant Staphylococcus aureus Carriers among patients and health care workers of a Tertiary care hospital in south of India. Indian Journal of Medical Microbiology, 2009; 27(1): 62-4.

8. Clinical and Laboratory Standards Institute. Performance standards for antimicrobial susceptibility testing; $27^{\text {th }}$ edition. CLSI document M100. Wayne, PA: Clinical and Laboratory Standards Institute; 2017.

9. Mohamed OA, Asma KE, Samira GA, Yousef MA. Cefoxitin Mannitol Salt Agar for Selective Isolation of Methicilin-Resistant Staphylococcus aureus. Ibnosina J. Med. BS, 2014; 6(1): 31-33. https://doi. org/10.4103/1947-489X.210354

10. Rajendra G, Soham G, Ujjwal N, Deepali A, Kesava $\mathrm{N}$, et al. Community prevalence of methicillin and vancomycin resistant Staphylococcus aureus in and around Bangalore, southern India. Revista da Sociedade Brasileira de Medicina Tropical, 2011; 44(3): 309-312. https://doi.org/10.1590/S003786822011005000035

11. Devjyoti M, Ankur B, Barnali P. Nasal carriage of methicillin resistant staphylococci in healthy population of east Sikkim. Indian Journal of Community Medicine, 2009; 34(4): 364- 365. https://doi.org/10.4103/09700218.58403

12. Chatterjee SS, Ray P, Aggarwal A, Das A and Sharma M. A community-based study on nasal carriage of Staphylococcus aureus. Indian J Med Res., 2009; 130: 742-748.

13. Reta A, Gedefaw L, Sewunet T, Beyene G. Nasal Carriage, Risk Factors and Antimicrobial Susceptibility Pattern of Methicillin Resistant Staphylococcus aureus among School Children in Ethiopia. J. Med. Microb. Diagn., 2015; 4(1): 177, 1-6. https://doi. org/10.4172/2161-0703.1000177

14. Rajendran SA, Narasimmal S, Sundaramoorthy VR, Alagappan U, Prabhu N and Ponniah T. Methicillin resistant Staphylococcus aureus - nasal carriage among health care workers. Journal of Global Biosciences, 2015; 4(6): 2518-2524. 